\title{
EJERCICIO FÍSICO Y CALIDAD DE VIDA EN ESTUDIANTES UNIVERSITARIOS
}

\author{
Alberto Paramio Leiva \\ Paloma Gil-Olarte Márquez \\ Cristina Guerrero Rodríguez \\ José M. Mestre Navas \\ Rocío Guil Bozal \\ Universidad de Cádiz \\ Email: alparamiole@gmail.com
}

https://doi.org/10.17060/ijodaep.2017.n1.v2.956

Fecha de Recepción: 1 Febrero 2017

Fecha de Admisión: 1 Abril 2017

\section{RESUMEN}

La recomendación del ejercicio físico como estrategia de prevención y promoción de la salud se ha establecido tanto en el ámbito sanitario como en el psicológico. A pesar del aumento del flujo de investigación en los últimos años, se es consciente de la necesidad de incrementar el respaldo empírico de las relaciones entre la actividad física y la calidad de vida. En favor de esta necesidad, este estudio se plantea como objetivos: 1. Clarificar si existen diferentes tipologías entre quienes realizan ejercicio físico y los que no. 2. Delimitar tipologías más específicas atendiendo a la frecuencia de realización desde una perspectiva de género. Para ello, se administró una batería de cuestionarios centrados los indicadores de calidad de vida: depresión, ansiedad estado, ansiedad rasgo, satisfacción personal y satisfacción con la vida a una muestra de 153 estudiantes universitarios. Mediante el análisis clúster biétapico, obtenemos 2 conglomerados entre los estudiantes que realizan alguna actividad física y aquellos estudiantes que no realizan ningún ejercicio físico, respondiendo los primeros a unas mejores puntuaciones en los indicadores anteriormente nombrados. Profundizando en la frecuencia, y teniendo en cuenta el género de los individuos que practican ejercicio, hemos diferenciado 4 conglomerados, 2 compuestos principalmente por hombres que se comportan como cabría esperar, respondiendo a una mejor calidad de vida el conglomerado que realiza una mayor frecuencia de ejercicio a la semana; y otras 2 compuestos por mujeres las cuales presentan diferencias que no se definen de forma tan pronunciada y algunas variables no se relacionan conforme a las expectativas. Este hecho remarca la necesidad de una investigación más exhaustiva. Animamos la labor de investigación en busca de elaborar programas de actividad física orientados a mejorar la calidad de vida en las distintas poblaciones.

Palabras clave: Ejercicio físico, calidad de vida, estudiantes, depresión, ansiedad, satisfacción personal, satisfacción con la vida. 


\section{EJERCICIO FÍSICO Y CALIDAD DE VIDA EN ESTUDIANTES UNIVERSITARIOS}

\section{ABSTRACT \\ Physical exercise and quality of life in university students}

The recommendation of physical exercise as a strategy for prevention and promotion of health has been established in both the health and psychological areas. Despite the increased flow of research in recent years, the need to increase the empirical support of the relationships between physical activity and quality of life is well known. In favor of this need, this study aims to: 1 . Clarify if there are different typologies between those who perform physical exercise and those who do not. 2. Delimit more specific typologies according to the frequency of realization from a gender perspective. To do this, a battery of questionnaires focused on quality of life indicators: depression, state anxiety (A-State), trait anxiety (A-Trait), personal satisfaction and satisfaction with life were administered to a sample of 153 university students. By means of the biphasic cluster analysis, we obtain 2 typologies among the students that perform some physical activity and those students who do not perform any physical exercise, the first responding to a better score in the previously mentioned indicators. Deepening the frequency, and taking into account the gender of individuals who exercise; we have differentiated 4 typologies, 2 composed mainly by men who behave as expected, responding to a better quality of life the category that performs a higher frequency of exercise a week; and 2 others composed of women who present differences that are not defined so pronounced and some variables are not related according to expectations. This fact underscores the need for more thorough investigation. We encourage research to develop physical activity programs aimed at improving the quality of life in different populations.

Key words: Physical exercise, quality of life, students. depression, anxiety, personal satisfaction, satisfaction with life.

\section{ANTECEDENTES}

La recomendación del ejercicio físico como técnica de prevención de enfermedades cardiovasculares y factor primordial en la promoción de la salud, se ha establecido de forma definitiva por parte de los profesionales sanitarios que proponen la actividad física moderada y una dieta equilibrada como principales factores de protección. Ya en los años ochenta, fue comprobado de forma concluyente que el ejercicio físico es parte primordial para el mantenimiento de una perspectiva de bienestar que engloba no sólo las variables biológicas, sino también los aspectos sociales y psicológicos. Desde los comienzos de la investigación de la actividad física, ya sea en forma de ejercicio o en la práctica deportiva, ésta comporta un factor decisivo para la conceptualización de la calidad de vida, reportando mejoras en el estado físico y psicológico (American College of Sport Medicine, 2000; Balaguer, Castillo \& Duda, 2008)

El ejercicio físico no sólo se ha postulado como una medida importante en el ámbito de la prevención y promoción de la salud, se ha comprobado experimentalmente su capacidad de acción para la mejora de los problemas tanto físicos como psicológicos (Seager, 2012; Ángeles, Jiménez, Sánchez \& Juan, 2016). Los constructos más estudiados en psicología, en su relación con el ejercicio físico, se encuentran relacionados con el estado de ánimo; entre ellos destacan la depresión, la ansiedad y la autoestima. Existen numerosos estudios con relación a estas variables.

Con respecto a la depresión, existen evidencias para determinar el efecto beneficioso que dispone la actividad física en esta dimensión (Martínez, Velázquez \& Castro, 2015). Este hecho se ha constatado en ambos sexos y todas las edades (Poblete-Valderrama, Castillo, Sandoval, Silva \& García, 2016). Estudios actuales reafirman el valor de un patrón de estilo de vida activo como factor de protección ante un aumento en el nivel de depresión (Gallegos-Carrillo et al., 2012).

En la reducción de los niveles de ansiedad, su eficacia se ha demostrado tanto en la ansiedad 
estado como rasgo (Martínez et al., 2015; Aznar, Curbelo \& Pérez, 2016). El efecto reductor de la ansiedad por el ejercicio físico se evidencia tanto en la población normal como en aquélla con trastornos psicopatológicos relacionados con esta dimensión. Además, se ha probado que su eficacia no reside tanto en su ocurrencia, sino en el tipo de actividad física que se realiza (Riahia, Haddada, Ouattasa \& Goebela, 2016). Sin embargo, Ios metaanálisis evidencian una pérdida de efectividad a medida que la edad de los sujetos aumenta. En una encuesta de la OMS del año 2016 (Stubbs et al., 2017), Ios análisis reportaron una relación clara entre un bajo nivel de actividad física y la prevalencia de problemas de ansiedad. También incidía en la necesidad de un estudio longitudinal para comprobar la direccionalidad de dicha correlación.

La relación entre la actividad física y la satisfacción personal y con la vida, ha sido mucho menos estudiadas que en el caso de la depresión o la ansiedad. Estos pocos estudios han revelado una correlación positiva entre ambas variables. En un reciente estudio para comprobar dicha relación en una muestra de estudiantes universitarios, los resultados mostraron que no existía una relación clara entre las variables, sin embargo, animaban a un estudio más exhaustivo del fenómeno (Busing \& West, 2016). En otros estudios de carácter longitudinal, la relación entre la actividad física y la satisfacción con la vida se hacía mucho más evidente en los adultos de mediana edad y los más ancianos, reportando estos una relación positiva, lo cual no se observó en los adultos jóvenes (Maher et al., 2015; Hernández, Jiménez \& Hernández, 2013).

Dentro de las principales características de las que debe disponer el ejercicio físico para reportar beneficios en el bienestar y la calidad de vida se encuentran:

Realizar la actividad física de forma óptima en la ejecución y de manera regular.

La intensidad de los ejercicios debe ser al menos moderada y mantenerse un mínimo de 20 minutos. Esto conlleva un estado óptimo de actividad dispuesta de entre 20 y 30 minutos de forma diaria 0 , al menos, tres días a la semana en días alternos

La programación del ejercicio debe desarrollarse progresivamente y desarrollarse y adaptarse a las características iniciales y posterior evolución de cada persona, variando así el tipo de ejercicio y la intensidad del mismo. Esto permitirá la adherencia al ejercicio de manera natural dentro de la rutina del individuo.

En un estudio realizado con 11.110 adolescente de diferentes países europeos se encontró una correlación estadísticamente significativa entre la frecuencia de ejercicio físico moderada de unos 60 minutos por sesión, con síntomas ansiosos y depresivos de forma negativa y con el bienestar de forma positiva (McMahon, et al., 2017).

No obstante, debemos tener en cuenta que ciertos componentes de la actividad física pueden generar efectos adversos y negativos cuando no se hace un uso responsable de la misma. La adicción a la práctica deportiva, la extrema competitividad, el carácter de trabajo obligatorio autoimpuesto, la presencia de violencia o la ausencia de diversión y disfrute, son factores de riesgo significativos en la adquisición de psicopatologías y enfermedades (Chirivella, 2001). Para evitar estos aspectos y sus respectivas consecuencias, se recomienda seguir una práctica deportiva saludable cumpliendo con las características anteriormente mencionadas.

La mayoría de los estudios reportan una mayor práctica de actividad física por parte de hombres que de mujeres. Siendo éstas más proclives a realizarlos por razones relacionadas con su salud (Busing \& West, 2016).

A la luz de estos resultados con la presente investigación nos planteamos realizar un estudio piloto que nos permita explorar si la realización de ejercicio físico constituye una variable que permita discriminar entre una o distintas agrupaciones naturales que identifiquen diversos grupos más o menos homogéneos en relación a diversos indicadores psicológicos de calidad de vida, y si la fre- 


\section{EJERCICIO FÍSICO Y CALIDAD DE VIDA EN ESTUDIANTES UNIVERSITARIOS}

cuencia con que se practica, y el género de quien lo practica, establece diferencias añadidas en una muestra de estudiantes de la Universidad de Cádiz .

\section{OBJETIVOS DE LA INVESTIGACIÓN}

1. Explorar la existencia de diferentes conglomerados o tipologías entre el alumnado de la Universidad de Cádiz que realiza, o no, ejercicio físico y diversos indicadores de calidad de vida. Concretamente niveles de Ansiedad Estado-Rasgo, de Depresión y de Satisfacción con la Vida y Satisfacción Personal.

2. Delimitar conglomerados o tipologías más específicas atendiendo a la frecuencia de realización de actividades deportivas y el sexo del alumnado de la Universidad de Cádiz respecto a los mismos indicadores de calidad.

\section{MUESTRA Y PARTICIPANTES}

El estudio fue realizado sobre una muestra de 153 estudiantes voluntarios la Universidad de Cádiz, compuesta por 110 mujeres (71.9\%) y 43 hombres (27,5\%). El 56,58\% cursaban estudios de Psicología, el 2I 39,47 \% de Relaciones Laborales y Recursos Humanos, el 1,32\% de del Grado de Maestros en Educación Infantil y el 2,63\% de Ciencias del Mar. Un 26,1\% cursaban su primer curso, el $34 \%$ estaban el segundo, el 5,9\% el tercero y el $44,4 \%$ el cuarto. La edad media fue 21,71 (rango 18-48).

\section{METODOLOGÍA E INSTRUMENTOS UTILIZADOS}

Para le realización de este estudio exploratorio se aplicó un diseño expo-facto observacional transversal. Se registraron las variables de interés y posteriormente se realizaron sendos análisis clúster bietápicos para poder conocer la existencia de posibles agrupaciones naturales 0 conglomerados que nos permitan diferenciar si dentro del alumnado encontramos diversas tipologías en relación a sus niveles percibidos de calidad de vida, la realización o no de ejercicio físico, la frecuencia de realización de tal ejercicio y el sexo. Las pruebas fueron administradas en horario de clase entre diversas asignaturas en las que el área de Psicología Social tenía responsabilidades docentes.

Para la evaluación de la Ansiedad Estado y Rasgo se utilizó el Cuestionario de Ansiedad EstadoRasgo (STAI) de Spielberger, Gorsuch y Lushene (1982). Esta escala evalúa dos conceptos diferentes de la ansiedad: La Ansiedad Estado, considerada como un estado emocional transitorio, caracterizado por sentimientos subjetivos, conscientemente percibidos, de atención y aprensión y por hiperactividad del sistema nervioso autónomo; y la Ansiedad Rasgo, que señala una propensión ansiosa, relativamente estable, que caracteriza a los individuos con tendencia a percibir las situaciones como amenazadoras.

Para evaluar la Depresión se utilizó el Inventario de Depresión de Beck-II (BDI-II) (Beck, Steer \& Brown, 1996), en su adaptación española de Sanz, Navarro y Vázquez (2003). Este inventario nos permite identificar y medir la gravedad de síntomas típicos de la depresión en adultos y adolescentes a partir de 13 años. Está adaptado a la población española.

Para evaluar la Satisfacción con la Vida se utilizó la Escala de Satisfacción con la Vida (SWLS) de Diener, Emmons, Larsen y Griffin (1985). Es un instrumento breve que nos permite evaluar los juicios cognitivos relacionados con la satisfacción con la vida y la satisfacción personal.

Para evaluar la Satisfacción Personal se utilizaron los ítems correspondientes a esta dimensión de la adaptación realizada por Novella (2002) de la Escala de Resiliencia de Wagnild y Young (1993). Esta nos permite evaluar y comprender el significado de la vida por parte del sujeto y cómo se contribuye a esta. 
Todos los cuestionarios son pruebas estandarizadas ampliamente utilizadas y con validez y fiabilidad ampliamente contrastadas.

Para el análisis de datos y la distribución de los distintos conglomerados, se ha utilizado el software informático IBM SPSS Statistics 24.

\section{RESULTADOS ALCANZADOS}

Para dar respuesta a nuestro primer objetivo y explorar el perfil, o los diversos perfiles, que podemos encontrar entre el alumnado de la Universidad de Cádiz en relación a sus niveles de calidad de vida percibida y la realización o no de ejercicio, realizamos un análisis de cluster bietápico introduciendo los valores de los criterios de calidad de vida ansiedad estado y rasgo, depresión, satisfacción perosnal y satisfacción con la vida y si realizaban ejercicio físico o no.

Fig, 1.

Resumen del modelo y el gráfico de calidad del clúster.

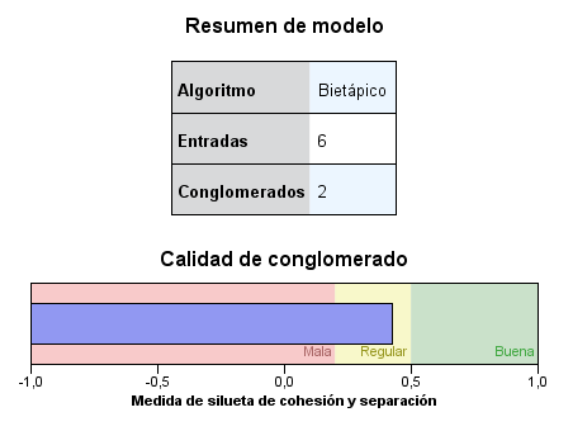

La Figura 1 muestra el resumen del modelo indicando que se han formado 2 clusters con las 6 características de entrada (variables categóricas y numéricas) seleccionadas y el gráfico de calidad del clúster indica que el resultado es correcto.

Fig, 2.

Comparación de los conglomerados en relación a la realización de ejercicio físico regular.

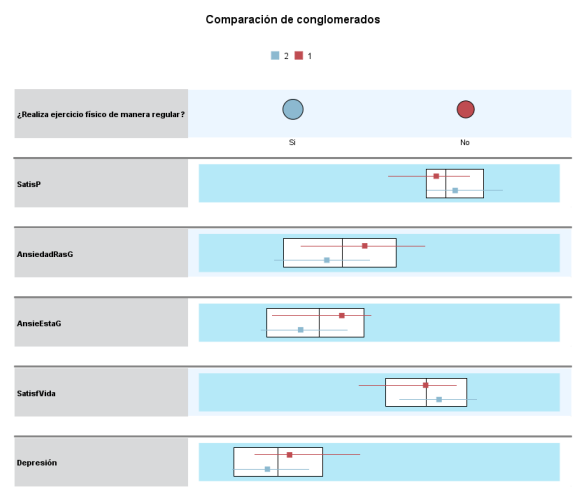




\section{EJERCICIO FÍSICO Y CALIDAD DE VIDA EN ESTUDIANTES UNIVERSITARIOS}

En la Figura 2 se muestran las características evaluadas en las filas y los conglomerados generados en las columnas, pudiendose entender mejor los factores de los que se componen los conglomerados, y mostrándese las diferencias entre ellos no sólo con respecto a los datos generales, sino entre sí. De esta manera comprobamos que el alumnado de la Universidad de Cádiz evaluado que realiza ejercicio, a diferencia de los que no lo realizan, presentan mayores niveles de satisfacción personal y con la vida, así como menores niveles de ansiedad rasgo y estado y de depresión, siendo la realización de jercicio el atributo de entrada que mayor importancia aporta en la formación de las agrupaciones.

Para dar respuesta a nuestro segundo objetivo se incluyen en el análisis de clústeres bietápicos la frecuencia de ejercicio físico y el sexo del alumnado. La Figura 3 muestra el resumen del modelo indicando que se han formado 4 clusters con las 7 características de entrada (variables categóricas y numéricas) seleccionadas y el gráfico de calidad del clúster indica que el resultado es correcto.

Fig, 3.

Resumen del modelo y el gráfico de calidad del clúster con las nuevas variables.

Resumen de modelo

\begin{tabular}{|l|l|}
\hline Algoritmo & Bietápico \\
\hline Entradas & 7 \\
\hline Conglomerados & 4 \\
\hline
\end{tabular}

Calidad de conglomerado

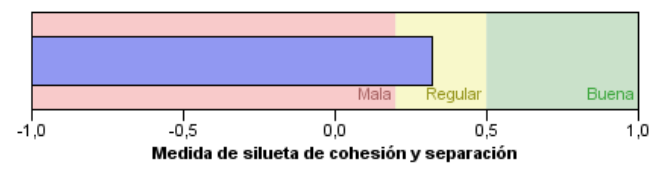

Para obtener una visualización de la distribución de los valores de cada campo de clúster, en la Figura 4 se muestran las distribuciones absolutas, así, observamos que el cluster número 1 lo constituyen el $21.1 \%$ de los sujetos, el segundo $23,3 \%$, el tercero el $21,1 \%$ y el cuarto el $34,4 \%$ siendo la frecuencia de realización de ejercicio la entrada con mayor importancia global en la creación del conglomerado, seguida por el sexo, la depresión, la ansiedad rasgo, la ansiedad estado y la satisfacción personal. 
Fig, 4.

Distribución de los conglomerados en cada una de las variables del estudio.

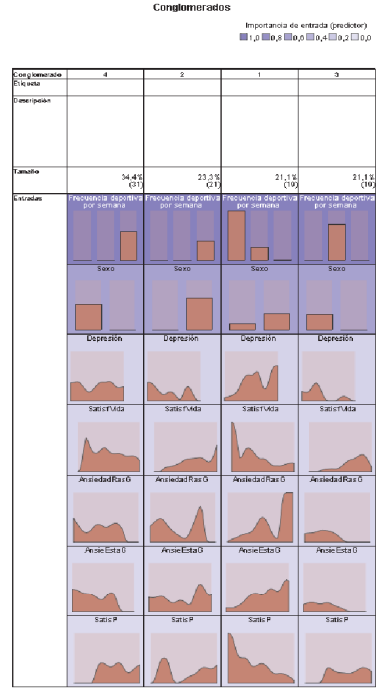

Fig, 5.

Comparación de los conglomerados en relación a la frecuencia del ejercicio físico y el sexo.

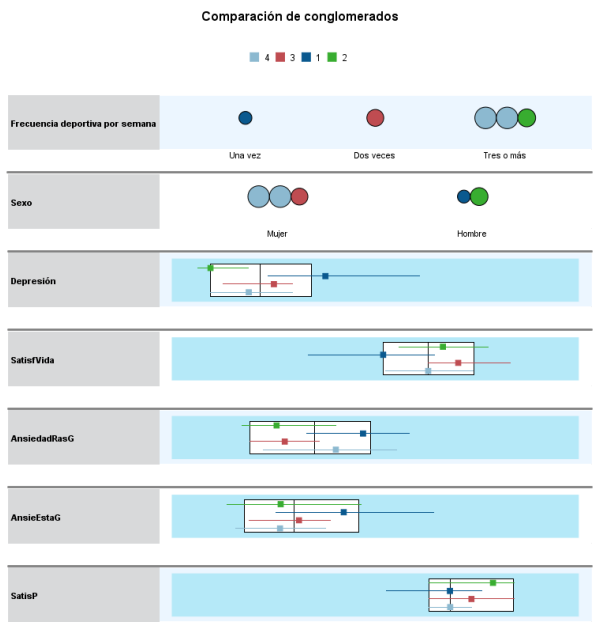

En la Figura 5 se muestran las características evaluadas en las filas y los conglomerados generados en las columnas, pudiendose comprobar la formación de cuatro tipologías formadas por alumnado: 


\section{EJERCICIO FÍSICO Y CALIDAD DE VIDA EN ESTUDIANTES UNIVERSITARIOS}

Conglomerado 1: Alumnos hombres que realiza ejercicio una vez por semana y presenta puntuaciones elevadas en depresión, ansiedad estado y rasgo, así como puntuación reducidas en satisfacción con la vida y en la media satisfacción personal.

Conglomerado 2: Alumnos hombres que realiza ejercicio tres veces o más por semana y presenta puntuaciones reducidas en depresión, ansiedad estado y rasgo y elevadas en satisfacción personal y por encima de la media en satisfacción con la vida.

Conglomerado 3: Alumnas mujeres que realiza ejercicio dos veces por semana y presenta puntuación por encima de la media en depresión, niveles medios en ansiedad estado, por debajo de la media en ansiedad rasgo y puntuación por encima de la media en satisfacción con la vida y satisfacción personal.

Conglomerado 4: Alumnas mujeres que realiza ejercicio tres veces o más por semana y que presenta puntuación por debajo de la media en depresión y ansiedad estado y bastante por encima de la media en rasgo. Puntuación en la media en satisfacción con la vida y satisfacción personal.

Como podemos observar, parece haber una marcada diferencia en función de la frecuencia con que se realiza ejercicio y el sexo del alumnado. En terminos generales, los que realizan ejercicio con más frecuencia paracen mostrar mejor calidad de vida que los que lo hacen con menos frecuencia. Dentro de los varones encontramos, no obstante, que aquellos que realizan deporte sólo una vez en semana son curiosamente los que presentan peores niveles de calidad de vida.

Los conglomerados constituidos por mujeres se mueven en niveles intermedios, siendo nuevamente las que más deporte realizan las que presentan mayores niveles de calidad de vida.

\section{DISCUSIÓN}

Los resultados obtenidos por los análisis de clústeres bietápicos nos permiten observar que la realización de ejercicio físico se acompaña de mejores niveles en los indicadores de calidad de vida evaluados, y que la frecuencia en la realización de ejercicio físico también parece establecer diferencias con estos niveles, así como en sexo de quien lo realiza. Los resultados indican que mientras más deporte se practique mejor calidad de vida, tanto en hombres como en mujeres, si bien la diferencia parece ser más marcada en el caso de los varones. Estos resultados refuerzan las conclusiones de aquellas investigaciones que situaban al ejercicio físico como un factor de protección determinante en el bienestar psicológico.

Igualmente parece confirmarse la idea de que la regularidad en el desarrollo de la actividad física y la duración del ejercicio son fundamentales a la hora de evaluar la importancia del mismo sobre los niveles de depresión, satisfacción personal y con la vida, así como con la ansiedad estado y rasgo. No obstante, para sacar conclusiones causales y predictoras se precisaría de estudios más amplios y longitudinales.

El primer conglomerado compuesto por hombres que realizan ejercicio una vez por semana, es el que se acompaña con las peores puntuaciones en las dimensiones de calidad de vida. Esto coincide con la idea de que no es sólo necesario la realización del ejercicio físico para obtener una mejor calidad de vida, sino que se requiere de una constancia y calidad del ejercicio para obtener resultados favorables. Muchos de estos estudiantes pueden haber estado realizando actividad física como estrategia de regulación para solventar las irregularidades mostradas en los resultados, pero debido a la falta de constancia no haber conseguido unos mejores niveles su calidad y bienestar de vida. Por otra parte, el conglomerado 2 (hombres con una frecuencia de ejercicio de tres o más veces a la semana) muestra una mejora considerable, lo que volvería a confirmar esta relación en sentido inverso, a mayor constancia en la práctica deportiva mejores resultados en la calidad de vida. Estos resultados nos animan a profundizar en esta relación en futuros trabajos. 
Los conglomerados compuestos principalmente por mujeres (Conglomerados 3 y 4), nos permiten distinguir matices diferentes. Los resultados no se comportan respecto a lo esperado en ciertas variables, aunque parecen mantener una coherencia respecto a la utilización del ejercicio físico como factor de protección para la salud. El conglomerado 4, pese a realizar ejercicio físico con mayor frecuencia que el conglomerado 3, obtiene peores resultados en ansiedad rasgo, satisfacción personal y satisfacción con la vida. Aquí nos surge la duda de si hacer más ejercicio es la causa de tener problemas de ansiedad e insatisfacción o es la consecuencia de ello. Es decir, si se busca el ejercicio como una estrategia de regulación del estado afectivo. Nuevamente consideramos necesario seguir explorando esta posible relación con estudios longitudinales, y con mayor muestra, para comprobar la dirección de estas relaciones así como su posible de carácter más explicativo y predictivo.

Es evidente que el ejercicio físico tiene un gran impacto sobre el estado de ánimo y la sensación de bienestar del individuo. Sin embargo, las características del mismo para resultar efectivo como estrategia de afrontamiento necesitan de un mayor apoyo empírico. Por lo tanto, animamos a que se siga investigando en otras muestran que faciliten la comprensión del beneficio de la práctica deportiva como estrategia de promoción y prevención de la salud tanto en el ámbito clínico como con poblaciones normalizadas.

\section{CONCLUSIÓN}

1. La realización o no de ejercicio físico se muestra como el predictor más importante en la determinación de agrupaciones naturales que muestran diferencias en el alumnado con mejores indicadores de calidad de vida de los que muestran valores más bajos, seguido de la satisfacción personal, ansiedad rasgo, ansiedad estado, satisfacción con la vida y depresión, con independencia de la frecuencia de la práctica y el sexo de la persona.

2. La frecuencia con la que se realiza ejercicio y el sexo de quien lo realiza se muestra a su vez como los predictor más importante en la determinación de agrupaciones naturales, seguidas de los niveles de depresión, satisfacción con la vida, ansiedad rasgo y estado, y satisfacción personal.

3. Potenciar el desarrollo de ejercicio físico en ambos sexos podría actuar como un factor de protección para la calidad de vida.

4. Se precisan estudios con muestras amplias en donde se pueda determinar la influencia del tipo de deporte practicado y la frecuencia en la calidad de vida, así como los procesos implicados en esta relación, siendo necesario atender a las diferencias de género.

\section{REFERENCIAS}

American College of Sports Medicine (2000). ACSM's guidelines for exercise testing and prescription (6th ed.). Philadelphia, PA: Lippincott, Williams \& Wilkins.

Ángeles, M. A. V., Jiménez, J. M., Sánchez, J. J. G., \& Juan, F. R. (2016). El efecto de un programa de ejercicios basado en Pilates sobre el estado de ánimo en adultos mayores mexicanos. Retos: nuevas tendencias en educación física, deporte y recreación, (30), 106-109.

Aznar, M. P. M., Curbelo, M. L., \& Pérez, L. G. (2016). Género, ejercicio físico y salud en personas mayores. En J. Soler, L. Aparicio, O. Díaz, E. Escolano \& A. Rodríguez (coord.). Inteligencia Emocional y Bienestar II: reflexiones, experiencias profesionales e investigaciones (pp. 598-611). Zaragoza: Ediciones Universidad de San Jorge.

Balaguer, I., Castillo, I., \& Duda, J. L. (2008). Apoyo a la autonomía, satisfacción de las necesidades, motivación y bienestar en deportistas de competición: un análisis de la teoría de la autodeterminación. Revista de Psicología del Deporte, 17(1), 123-139. 


\section{EJERCICIO FÍSICO Y CALIDAD DE VIDA EN ESTUDIANTES UNIVERSITARIOS}

Beck, A. T., Steer, R. A., \& Brown, G. K. (1996). Manual for the Beck depression inventory-II. San Antonio, TX: Psychological Corporation.

Busing, K., \& West, C. (2016). Determining the Relationship Between Physical Fitness, Gender, and Life Satisfaction. SAGE Open, 6(4), 2158244016669974.

Chirivella, E. C. (2001). Deporte, salud, bienestar y calidad de vida. Cuadernos de psicología del deporte, 1.

Diener, E., Emmons, R., Larsen, R. J. \& Griffin, S. (1985). The Satisfaction With Life Scale. Journal of Personality Assessment, 49(1), 71-75.

Gallegos-Carrillo, K., Flores, Y. N., Denova-Gutiérrez, E., Méndez-Hernández, P., DosamantesCarrasco, L. D., Henao-Morán, S., ... \& Salmerón, J. (2013). Physical activity and reduced risk of depression: Results of a longitudinal study of Mexican adults. Health Psychology, 32(6), 609.

Hernández, E. D. L. C. R., Jiménez, E. S., \& Hernández, N. R. (2013). El ejercicio físico, una alternativa para mejorar la calidad de vida en el adulto mayor sedentario. Revista Cubana de Tecnología de la Salud, 3(3).

Maher, J. P., Pincus, A. L., Ram, N., \& Conroy, D. E. (2015). Daily physical activity and life satisfaction across adulthood. Developmental psychology,, 51(10), 1407-1419.

Martínez, B. I., Velázquez, I. 0., \& Castro, M. J. G. (2015). Prevalencia de realización y prescripción de ejercicio físico en pacientes diagnosticados de ansiedad y depresión. Atención Primaria, 47(7), 428-437.

McMahon, E. M., Corcoran, P., O’Regan, G., Keeley, H., Cannon, M., Carli, V., ... \& Balazs, J. (2017). Physical activity in European adolescents and associations with anxiety, depression and well-being. European child \& adolescent psychiatry, 26(1), 111-122.

Novella, A. (2002). Incremento de la resiliencia luego de la aplicación de un programa de psicoterapia breve en madres adolescentes. (Tesis para optar el grado de Magíster en Psicología, mención en Psicología clínica y de la salud). Universidad Nacional Mayor de San Marcos, Lima, Perú.

Riahia, M. A., Haddada, M., Ouattasa, A., \& Goebela, R. (2016). The Moderating Effect of Physical Exercise in Anxiety Disorder: A review.

Poblete-Valderrama, F., Castillo, C. M., Sandoval, E. D., Silva, P. V., \& García, M. A. (2016). Depresión, cognición y calidad de vida en adultos mayores activos. Revista Ciencias de la Actividad Física, 16(2).

Sanz, J., Navarro, M.E. \& Vázquez, C. (2003). Adaptación española del Inventario para la Depresión de Beck-II (BDI-II): Propiedades psicométricas en estudiantes universitarios. Análisis y Modificación de Conducta, 29(124), 239-288.

Seager, M. (2012). Towards a public health psychology. Journal Public Mental Health, 11(3), 153-156.

Spielberger, C.D., Gorsuch, R.L., \& Lushene, R. (1982). Manual del Cuestionario de Ansiedad Estado/Rasgo (STAI). Madrid, España: TEA Ediciones.

Stubbs, B., Koyanagi, A., Hallgren, M., Firth, J., Richards, J., Schuch, F., ... \& Vancampfort, D. (2017). Physical activity and anxiety: A perspective from the World Health Survey. Journal of Affective Disorders, 208, 545-552.

Wagnild, G. \& Young, H. (1993). Development and psychometric evaluation of the resilience scale. Journal of Nursing Measurement, 1(2), 165-167. 